\title{
The Utility of Drug Challenge Testing in Brugada Syndrome: a Systematic Review and Meta-analysis
}

\author{
Pattara Rattanawong ${ }^{1}$, Jakrin Kewcharoen ${ }^{2}$, Chanavuth Kanitsoraphan ${ }^{2}$, Wasawat \\ Vutthikraivit ${ }^{3}$, Prapaipan Putthapiban ${ }^{4}$, Narut Prasitlumku ${ }^{5}$, Poemlarp Mekraksakit ${ }^{6}$, \\ Raktham Mekritthikrai ${ }^{2}$, and Eugene Chung ${ }^{7}$ \\ ${ }^{1}$ Mayo Clinic Arizona \\ ${ }^{2}$ University of Hawaii Internal Medicine Residency Program \\ ${ }^{3}$ Texas Tech University Health Sciences Center \\ ${ }^{4}$ Albert Einstein Medical Center \\ ${ }^{5}$ University of Hawaii Internal Medicine Residency Pr \\ ${ }^{6}$ Phramongkutklao College of Medicine \\ ${ }^{7}$ University of Michigan Health System
}

April 27, 2020

\begin{abstract}
Introduction: Brugada syndrome is associated with ventricular arrhythmia leading to sudden cardiac death. Risk stratification is challenging, as major arrhythmic events (MAE) are rare. We assessed the utility of drug challenge testing in Brugada syndrome by a systematic review and meta-analysis. Methods and results: We comprehensively searched the databases of MEDLINE and EMBASE from inception to May 2019. Included studies compared the incidence of MAE between spontaneous and drug challenge induced Type-1. Data were combined using the random-effects, generic inverse variance method, to calculate pooled incidence and odds ratio (OR). Mixed-effects Poisson regression was used to calculated incidence rate ratio (IRR). Eighteen studies from 2006 to 2018 were included (4,099 patients, mean follow-up 4.5 years). Pooled annual incidences of MAE in spontaneous, drug challenge induced (regardless of symptoms), asymptomatic drug challenge induced, and symptomatic drug challenge induced Type-1 were 23.8 (95\% confidence interval [CI]: 19.8-27.8), 6.5 (95\% CI: 3.9-9.1), 2.1 (95\% CI: -0.3-4.4), and 19.6 (95\% CI: 9.9-29.3) per 1,000 person-years respectively. The incidence of MAE between symptomatic drug challenge induced and asymptomatic spontaneous Type-1 was not statistically different (IRR=1.0, 95\%CI: 0.6-1.7). The presence of ventricular tachyarrhythmia during drug challenge testing was a predictor of MAE (OR=3.73, 95\% CI: 1.77-7.86, $\mathrm{p}=0.001)$. Conclusions: The incidence of MAE in drug challenge induced Type-1 in asymptomatic patients is low. The incidence of MAE between symptomatic drug challenge induced and asymptomatic spontaneous Type-1 was similar. Ventricular tachyarrhythmia during drug challenge testing could be a useful risk marker for MAE in Brugada syndrome.
\end{abstract}

The Utility of Drug Challenge Testing in Brugada Syndrome: a Systematic Review and Metaanalysis

Pattara Rattanawong, $\mathrm{MD}^{1,2}$, Jakrin Kewcharoen, $\mathrm{MD}^{3}$, Chanavuth Kanitsoraphan, $\mathrm{MD}^{3}$, Wasawat Vutthikraivit, $\mathrm{MD}^{4}$, Prapaipan Puttapiban, $\mathrm{MD}^{5}$, Narut Prasitlumkum, $\mathrm{MD}^{3}$, Poemlarp Mekraksakit, $\mathrm{MD}^{4}$, Raktham Mekrittikrai, $\mathrm{MD}^{3}$, Eugene $\mathrm{H}$ Chung $\mathrm{MD}^{6}$

1) Department of Cardiovascular Medicine, Mayo Clinic, Phoenix, AZ, USA

2) Faculty of Medicine Ramathibodi Hospital, Mahidol University, Bangkok, Thailand 
3) University of Hawaii Internal Medicine Residency Program, Honolulu, HI, USA

4) Department of Internal Medicine, Texas Tech University Health Sciences Center, Lubbock, TX, USA

5) Department of Medicine, Einstein Medical Center, Philadelphia, PA, USA.

6) Department of Internal Medicine, Michigan Medicine, University of Michigan, Ann Arbor, Michigan

Running head: Drug challenge testing in Brugada syndrome

Word count : 3,274

Financial Support : This research did not receive any specific grant from funding agencies in the public, commercial, or not-for-profit sectors.

Declaration of Interest : None

Address for correspondence:

Pattara Rattanawong, MD

Department of Cardiovascular Medicine

Mayo Clinic 5777 East Mayo Boulevard Phoenix, AZ, USA, 85054

Phone: 808-859-3848 Email: Rattanawong.pattara@mayo.edu

Introduction: Brugada syndrome is associated with ventricular arrhythmia leading to sudden cardiac death. Risk stratification is challenging, as major arrhythmic events (MAE) are rare. We assessed the utility of drug challenge testing in Brugada syndrome by a systematic review and meta-analysis.

Methods and results: We comprehensively searched the databases of MEDLINE and EMBASE from inception to May 2019. Included studies compared the incidence of MAE between spontaneous and drug challenge induced Type-1. Data were combined using the random-effects, generic inverse variance method, to calculate pooled incidence and odds ratio (OR). Mixed-effects Poisson regression was used to calculated incidence rate ratio (IRR). Eighteen studies from 2006 to 2018 were included (4,099 patients, mean followup 4.5 years). Pooled annual incidences of MAE in spontaneous, drug challenge induced (regardless of symptoms), asymptomatic drug challenge induced, and symptomatic drug challenge induced Type-1 were 23.8 (95\% confidence interval [CI]: 19.8-27.8), 6.5 (95\% CI: 3.9-9.1), 2.1 (95\% CI: -0.3-4.4), and 19.6 (95\% CI: 9.9-29.3) per 1,000 person-years respectively. The incidence of MAE between symptomatic drug challenge induced and asymptomatic spontaneous Type-1 was not statistically different (IRR=1.0, 95\%CI: 0.6-1.7). The presence of ventricular tachyarrhythmia during drug challenge testing was a predictor of MAE $(\mathrm{OR}=3.73$, 95\% CI: 1.77-7.86, $\mathrm{p}=0.001)$.

Conclusions: The incidence of MAE in drug challenge induced Type-1 in asymptomatic patients is low. The incidence of MAE between symptomatic drug challenge induced and asymptomatic spontaneous Type-1 was similar. Ventricular tachyarrhythmia during drug challenge testing could be a useful risk marker for MAE in Brugada syndrome.

Keywords: Drug challenge testing, Brugada syndrome, Sodium channel blocker

\section{Abbreviations}

BrS Brugada syndrome

VF ventricular fibrillation

SCD sudden cardiac death

MAE major arrhythmic events

SCB sodium channel blocker 
ECG electrocardiogram

NOS Newcastle-Ottawa quality assessment scale

OR odds ratio

IRR Incidence rate ratio

CI confidence interval

ICD implantable cardioverter defibrillator

\section{Introduction}

Brugada syndrome (BrS) was first described as a clinical syndrome in 1992 and predisposes patients to ventricular fibrillation (VF), premature sudden arrhythmic death syndrome, and aborted sudden cardiac death (SCD). Typically, patients present in the third or fourth decade of life. Even though most patients are asymptomatic at the time of diagnosis (approximately 63\%), major arrhythmic events (MAE) can develop at a rate of $12 \%$ over 10 years (1-3). The disease is most prevalent in Southeast Asia where the prevalence has been reported as 3.7 per 1,000 and up to 17.7 per 1,000 in Thailand $(4,5)$.

Drug challenge testing is a common provocation test performed to unmask Type- 1 Brugada pattern in patients with suspected BrS. In the past, a drug challenge induced Type-1 Brugada pattern was considered diagnostic of BrS (6). However, the "Shanghai Score System" was recently proposed, and in that, a drug challenge induced Type-1 Brugada pattern is less emphasized and scores as one component of the diagnostic criteria for BrS (1). That said, previous studies have suggested that positive drug challenge testing is associated with MAE (6). There are conflicting data on the incidence of MAE after drug challenge testing and the utilization of drug challenge testing. We sought to systematically review the incidence and the utility of drug challenge testing in the management of patients with possible BrS.

\section{Methods}

\subsection{Search strategy}

Two investigators (WV and PP) independently searched for published studies indexed in MEDLINE and EMBASE databases from inception to August 2019 using a search strategy that including the terms "sodium channel blocker", "ajmaline", "pilsicainide", "flecainide", "procainamide", and "Brugada syndrome" as described in supplementary file 1. Only full articles in English and studies conducted in cohorts were included. A manual search for additional pertinent studies and review articles using references from retrieved articles was also completed.

\subsection{Inclusion criteria}

The eligibility criteria included the following: (1) Cohort studies (prospective or retrospective) or randomized control trials reporting MAE after the diagnosis of BrS including SCD, sudden cardiac arrest, VF, sustained ventricular tachycardia, and appropriate shock in $\mathrm{BrS}$ patients diagnosed from drug challenge test by sodium channel blocker (SCB) and spontaneous Type-1 electrocardiogram (ECG).

(2) Adjusted or unadjusted relative risk, Odds ratio (OR), hazard ratio with $95 \%$ confidence interval (CI), or sufficient raw data for the calculation were provided. Patients with spontaneous Type-1 ECG were used as controls.

Study eligibility was independently determined by two investigators (CK and NP) and differences were resolved by mutual consensus. The Newcastle-Ottawa quality assessment scale (NOS) was used to assess each study's quality in three domains, recruitment and selection of the participants, similarity and comparability between the groups, and ascertainment of the outcome of interest among cohort and case-control studies (7).

2.3 Data extraction A standardized data collection form was used to obtain the following information from each study: title of study, name of first author, year of publication, study design, country of origin, number, 
gender and age of the participants, Brugada ECG pattern, incidence of MAE, available MAE outcome, follow-up duration, and confounders that were adjusted in the multivariable analysis, if available. Two investigators (JK and PM) independently performed this data extraction process to ensure accurate data extraction. Any data discrepancy was resolved by referring back to the original articles.

\subsection{Definitions}

\section{Brugada syndrome and Drug Challenge Testing}

Brugada syndrome was diagnosed in patients with ST-segment elevation with type 1 morphology [?]2 $\mathrm{mm}$ in [?]1 lead in the right precordial leads V1, V2, positioned in the 2nd, 3rd, or 4th intercostal space occurring either spontaneously or immediately after drug challenge testing with intravenous administration of SCB class I antiarrhythmic drugs (6). Asymptomatic Brugada syndrome was defined as Brugada syndrome without history of syncope or documented SCD, sudden cardiac arrest, VF, sustained ventricular tachycardia. Symptomatic Brugada syndrome was defined as Brugada syndrome with history of syncope or documented $\mathrm{SCD}$, sudden cardiac arrest, VF, sustained ventricular tachycardia (6).

\section{Major arrhythmic events}

MAE were defined as any of the following: SCD, sudden cardiac arrest, VF, sustained ventricular tachycardia, or implantable cardioverter defibrillator (ICD) appropriate shock. SCD was defined as an unexpected, nontraumatic death that occurred within $60 \mathrm{~min}$ from the onset of new or worsening symptoms or within 24 hours of last being observed alive and well (8). Sudden cardiac arrest was defined as a sudden cessation of cardiac activity with hemodynamic collapse for which an intervention or spontaneous reversion restored spontaneous circulation.

VF was defined as documented VF rhythm from standard 12-lead ECG or ECG monitoring, or as defined in each study. Sustained ventricular tachycardia was defined as a sustained organized ventricular rhythm, documented from standard 12-lead ECG or ECG monitoring, faster than 100 beats per minute lasting at least 30 seconds or requiring termination earlier due to hemodynamic instability. Only sustained ventricular tachycardia, VF, and appropriate ICD shocks were counted in this study. Non-sustained ventricular tachycardia and inappropriate shock were not considered an outcome of interest. Ventricular tachyarrhythmias are defined as documented VF rhythm or sustained ventricular tachycardia.

\subsection{Statistical analysis}

We performed a meta-analysis of the included studies using a random-effects model. Studies were excluded if they did not include an outcome in each intervention group, did not have enough information required for continuous data comparison, or shared the same patient population. Incidence rate ratios (IRR) of events were calculated by dividing the number of incident cases of events by the total number of person-years follow-up. IRR were pooled after applying the Freeman-Turkey double arcsine transformation to stabilize the variances (9). We pooled the point estimates of IRR, odds ratio, and incidence rate from each study using the generic inverse-variance method of Der Simonian and Laird (10). If no event was observed, pooled IRR were estimated using Poisson regression with random intervention effects (11). The heterogeneity of effect size estimates across these studies was quantified using the $\mathrm{I}^{2}$ statistic. The $\mathrm{I}^{2}$ statistic ranges in value from 0 to $100 \%$ ( $\mathrm{I}^{2}<25 \%$, low heterogeneity; $\mathrm{I}^{2}=25 \%-50 \%$, moderate heterogeneity; and $\mathrm{I}^{2}>50 \%$, substantial heterogeneity). A sensitivity analysis was performed to assess the influence of the individual studies on the overall results by omitting one study at a time. Publication bias was assessed using a funnel plot and the Egger's regression test $(12)$. ( $\mathrm{p}<0.05$ was considered significant). All data analyses were performed using the STATA SE version 14.2.

\subsection{Sensitivity analysis}

We used a sequential exclusion strategy, as described by Patsopoulos et al., to examine whether overall estimates were influenced by the substantial heterogeneity observed (13). We sequentially and cumulatively 
excluded studies that accounted for the largest share of heterogeneity until $\mathrm{I}^{2}$ was less than $50 \%$. We then examined whether RR estimates were consistent.

\section{Results}

3.1 Search results Our search strategy yielded 815 potentially relevant articles (465 articles from EMBASE and 350 articles from MEDLINE). After the exclusion of duplicated articles, 732 articles underwent title and abstract review. At this stage, 633 articles were excluded as they were not cohort, case-control, or randomized controlled trials, were not conducted in BrS patients or the titles and abstracts were not relevant. This left 99 articles for full-length review. Another 81 studies were excluded as they did not report data regarding SCB use, outcome of interest, or did not provide sufficient data to calculate OR. Therefore, a total of 18 studies were included in this meta-analysis $(3,14-30)$. Figure 1 outlines the search and literature review process.

\subsection{Description of included studies}

Eighteen studies from 2006 to 2018 were included in this meta-analysis involving 4,099 Brugada syndrome patients. Seventeen of 18 studies involving 3,596 Brugada syndrome patients reported the incidences of MAE in spontaneous Type- 1 and drug challenge induced Type-1 Brugada pattern (3, 14-26, 28-30). Three of eighteen studies involving 776 Brugada syndrome patients reported MAE during drug challenge testing $(16,27,30)$. Six of eighteen studies involving 541 Brugada syndrome patients reported symptomatic versus asymptomatic patients who had undergone drug challenge testing $(3,14,21,23,24,30)$. Mean follow-up was 4.5 years and $57.6 \%$ of Brugada syndrome patients were diagnosed by drug challenge induced Type- 1 Brugada pattern. A summary of study characteristics is shown in Table 1.

3.3 Quality assessment of included studies The NOS of included studies are described in Supplement Table. The NOS uses a star system (0 to 9 ) to evaluate included studies on 3 domains: selection, comparability, and outcomes. Higher scores represent a higher study quality.

\subsection{Meta-analysis results}

Pooled annual incidences of MAE in spontaneous and drug challenge induced Type-1 Brugada pattern were 23.8 (95\% CI: 19.8-27.8) (Figure 2) and 6.5 (95\% CI: 3.9-9.1) per 1,000 person-years (Figure 3) respectively. Spontaneous Type-1 Brugada pattern was associated with a higher risk of MAE (OR=3.05, 95\%CI: 2.08-4.46, $\mathrm{p}<0.001, \mathrm{I}^{2}=23.8 \%$ ) than drug challenge induced Type-1 Brugada pattern (Figure 4).

We performed subgroup analysis of symptomatic (with history of syncope or documented SCD, sudden cardiac arrest, VF, sustained ventricular tachycardia) and asymptomatic (without history of syncope or documented SCD, sudden cardiac arrest, VF, sustained ventricular tachycardia) drug challenge induced Type-1 Brugada pattern subjects. In asymptomatic individuals with drug challenge induced Type-1 Brugada pattern, pooled annual incidences of MAE was 2.1 (95\% CI: -0.3-4.4) per 1,000. In symptomatic individuals with drug challenge induced Type-1 Brugada pattern, pooled annual incidences of MAE was 19.6 (95\% CI: 9.9-29.3) per 1,000. Thus, the combination of symptoms and drug challenge induced Type-1 Brugada pattern was associated with a higher risk of MAE (IRR=9.7, 95\% CI: 3.6-25.9, $\mathrm{p}<0.001$ ) than drug challenge induced Type-1 Brugada pattern but no history of symptoms. Drug challenge induced Type-1 Brugada pattern in symptomatic patients has a similar incidence of MAE when compared to asymptomatic spontaneous Type-1 Brugada pattern (IRR=1.0, 95\%CI: 0.6-1.7, $\mathrm{p}=0.861)$.

The presence of ventricular tachyarrhythmia during drug challenge testing was associated with four-fold increased risk of MAE in drug challenge induced $\mathrm{BrS}$ patients (pooled $\mathrm{OR}=3.73,95 \%$ CI: 1.77-7.86, $\mathrm{p}=0.001$, $\mathrm{I}^{2}=0.0 \%$ ) (Figure 5) and increased to 13 -fold in the subgroup of symptomatic patients (pooled $\mathrm{OR}=12.9$, $95 \%$ CI: 3.8-43.2, $\left.\mathrm{p}<0.001, \mathrm{I}^{2}=0.0 \%\right)$. In asymptomatic drug challenge induced BrS patients, ventricular tachyarrhythmia during drug challenge testing increased risk of MAE but non-significant $(\mathrm{OR}=2.78,95 \%$ CI: 0.07-103.83) (data only available from one study)(16).

\subsection{Sensitivity analysis}


To assess the stability of the results of the meta-analysis, we conducted a sensitivity analysis for each outcome by excluding one study at a time. For every outcome, none of the results were significantly altered, as the results after removing one study at a time were similar to that of the main meta-analysis indicating that our results were robust.

\subsection{Publication bias}

To investigate potential publication bias of the association of a spontaneous Type- 1 Brugada pattern and increased risk of MAE, we examined the funnel plot of the included studies in assessing change in log OR of MAE (Figure 6). The vertical axis represents study size (standard error) while the horizontal axis represents effect size $(\log \mathrm{OR})$. From this plot, no bias was observed because distribution of studies was symmetrical on both sides of the mean. Egger's test showed no small study bias $(p=0.688)$.

Only four studies included in this meta-analysis could be used to assess the association between ventricular tachyarrhythmia during drug challenge testing and increased risk of MAE. Thus, we did not perform a funnel plot or Egger's test (12).

\section{Discussion}

A main finding from this meta-analysis is that the pooled annual incidences of MAE in subjects with spontaneous Type-1 Brugada pattern was 4-fold higher than the pooled annual incidences of MAE in those with drug challenge induced Type-1 Brugada pattern. Another important finding was that the annual incidences of MAE in drug challenge induced Type-1 Brugada pattern in asymptomatic patients was as low as 2.1 per 1000 person-years. However, drug challenge induced Type-1 Brugada pattern in symptomatic patients has similar incidence of MAE when compared to spontaneous Type-1 Brugada pattern (IRR=1.0). The utilization of drug challenge induced Type-1 Brugada pattern for diagnosing Brugada syndrome in asymptomatic patient is limited. However, the presence of ventricular tachyarrhythmia during drug challenge testing was an independent predictor of MAE in Brugada syndrome patients.

Brugada syndrome has been approximated to be the cause in $4 \%$ of all SCD and $20 \%$ of SCD in patients with structurally normal hearts (2). Implantable cardioverter defibrillator (ICD) implantation is a class I recommendation in BrS patients with a documented history of MAE (31). However, a majority of newly diagnosed BrS patients, especially those with a drug challenge induced Type-1 Brugada pattern, have no previous history of MAE (32). It thus remains a challenge to identify asymptomatic patients who are at risk for MAE and therefore might benefit from an ICD (31).

Disopyramide and procainamide were the first two SCB reported to transiently slow down conduction and repolarization in $\operatorname{BrS}(33)$. Ajmaline $(1 \mathrm{mg} / \mathrm{kg})$, procainamide $(10 \mathrm{mg} / \mathrm{kg})$, and flecainide $(2 \mathrm{mg} / \mathrm{kg})$ were later tested as provocative drugs in BrS evaluation. Their sensitivity for unmasking Type-1 Brugada pattern was found to be at $100 \%$ and positive results were $100 \%$ in a small study $(n=34)$. Drug challenged test provoked Type-1 Brugada pattern in all patients $(n=11)$ who had sodium channel mutation without baseline ST elevation ECG (34). Positive drug challenge testing is widely used and considered in current guidelines as diagnostic of BrS regardless of symptoms (6). However, drug challenge testing may over-diagnose BrS. Most BrS patients (approximately 70\%) in Europe were diagnosed with asymptomatic Brugada syndrome but positive ajmaline test (3). Ajmaline was more likely to provoke Type-1 Brugada pattern than procainamide but the false-positive results of Ajmaline was undetermined (35). A BrS syndrome diagnosis criteria "Shanghai Score System" was proposed in the recent J-Wave Syndrome Expert Consensus Conference Report to assign fewer points to drug challenge induced Type-1 Brugada pattern than spontaneous Type-1 Brugada pattern (2.0 VS 3.5 points respectively) (1).

Our study results are in line with the recent J-Wave Syndrome Expert Consensus Conference in that SCB drug challenge testing may over-diagnose BrS. The pooled annual incidences of MAE in drug challenge induced Type-1 Brugada pattern subjects is only 6.1 per 1000 person-years and only 2.1 per 1000 personyears in asymptomatic patients who had drug challenge induced Type-1 pattern, compared to 23.5 per 1000 person-years in spontaneous Type-1 Brugada pattern. The utilization of drug challenge induced Type-1 
Brugada pattern for diagnosing Brugada syndrome in asymptomatic patients is therefore limited. However, drug challenge induced Type-1 Brugada pattern in symptomatic patients has a similar incidence of MAE when compared to asymptomatic spontaneous Type- 1 Brugada pattern in our pooled analysis $(\mathrm{IRR}=1.0)$. In the FINGER registry, the incidence of MAE in asymptomatic BrS patients was very low at 5 per 1000 person-years as well (3). In 10 years of follow-up in the BrS ICD registry, the incidence of appropriate shock was 48 per 1000 person-years in patients with aborted sudden cardiac arrest, 19 per 1000 person-years for BrS patients with syncope, and only 12 per 1000 person-years for those who were asymptomatic at implantation (36). Recent meta-analysis of clinical outcomes after ICD implantation in BrS showed high incidence of ICDrelated complications, including inappropriate shocks 33 per 1000 person-years, lead malfunction 16 per 1000 person-years, lead dislocation, 6 per 1000 person-years lead dislocation 4 per 1000 person-years (37). The lower annual incidence of MAE in drug challenge induced Type-1 Brugada pattern versus the lower incidence of ICD related complications should be taken into account when considering ICD implantation in patients.

The presence of ventricular tachyarrhythmia during drug challenge testing was an independent predictor of MAE in BrS patients. There has been disagreement between studies regarding the association between ventricular tachyarrhythmia during drug challenge testing and risk of MAE in BrS patients. However, our pooled OR from 4 studies suggested that ventricular tachyarrhythmia during drug challenge testing could be a useful marker of increased risk of MAE in patients with BrS, especially in asymptomatic patients. We also demonstrated that spontaneous Type-1 Brugada pattern was associated with a higher risk of MAE than drug challenge induced Type-1 Brugada pattern, similar to previous studies.

\section{Limitations}

Our study is not without limitations. First, we were not able to address sensitivity and specificity of drug challenge testing by meta-analysis because of insufficient data provided in included articles. Secondly, four different SCB were used across the studies- likely the main source of cause heterogeneity in our results (SCB and doses are shown in Table 1). Moreover, for the pooled OR of ventricular tachyarrhythmia during drug challenge testing, extracted data from the included studies were not all adjusted for other variables and may be influenced by other confounders.

\section{Conclusions and Clinical Implications}

Drug challenge induced Type-1 Brugada pattern in symptomatic patients has similar incidence of MAE when compared to spontaneous Type-1 Brugada pattern. However, the incidence of MAE in drug challenge induced Type-1 Brugada pattern in asymptomatic patients is very low. A positive drug challenge testing is not a good predictor of MAE in asymptomatic patient. Ventricular tachyarrhythmia during drug challenge testing could be a useful marker of risk for MAE in patients with Brugada syndrome.

Table and figure legends

Figure 1 : Search methodology and selection process.

Figure 2:Forest plot of the pooled incidence of MAE in spontaneous Type-1 BrS patients.

Figure 3: Forest plot of the pooled incidence of MAE in drug challenge induced Type-1 BrS patients.

Figure 4: Forest plot demonstrating the association of a spontaneous Type-1 Brugada pattern and increased risk of MAE when compared to drug challenge induced Type-1 Brugada pattern.

Figure 5: Forest plot demonstrating the association of ventricular tachyarrhythmia during drug challenge testing and increased risk of MAE in drug challenge induced Type-1 BrS patients.

Figure 6: Funnel plot of meta-analyis of demonstrating the association of a spontaneous Type-1 Brugada pattern and increased risk of MAE when compared to drug challenge induced Type-1 Brugada pattern.

Table 1:Summary characteristics of individual included studies of patients with a Brugada syndrome .

Supplementary file 1 : Search strategy 
Supplementary file 2: Supplement Table (Newcastle-Ottawa quality assessment scale of included studies in meta-analysis).

\section{Reference}

1. Antzelevitch C, Yan GX, Ackerman MJ, Borggrefe M, Corrado D, Guo J, et al. J-Wave syndromes expert consensus conference report: Emerging concepts and gaps in knowledge. Heart Rhythm. 2016;13(10):e295324.

2. Brugada J, Campuzano O, Arbelo E, Sarquella-Brugada G, Brugada R. Present Status of Brugada Syndrome: JACC State-of-the-Art Review. J Am Coll Cardiol. 2018;72(9):1046-59.

3. Probst V, Veltmann C, Eckardt L, Meregalli PG, Gaita F, Tan HL, et al. Long-term prognosis of patients diagnosed with Brugada syndrome: Results from the FINGER Brugada Syndrome Registry. Circulation. 2010;121(5):635-43.

4. Vutthikraivit W, Rattanawong P, Putthapiban P, Sukhumthammarat W, Vathesatogkit P, Ngarmukos T, et al. Worldwide Prevalence of Brugada Syndrome: A Systematic Review and Meta-Analysis. Acta Cardiol Sin. 2018;34(3):267-77.

5. Rattanawong P, Ngarmukos T, Chung EH, Vutthikraivit W, Putthapiban P, Sukhumthammarat W, et al. Prevalence of Brugada ECG Pattern in Thailand From a Population-Based Cohort Study. J Am Coll Cardiol. 2017;69(10):1355-6.

6. Priori SG, Wilde AA, Horie M, Cho Y, Behr ER, Berul C, et al. HRS/EHRA/APHRS expert consensus statement on the diagnosis and management of patients with inherited primary arrhythmia syndromes: document endorsed by HRS, EHRA, and APHRS in May 2013 and by ACCF, AHA, PACES, and AEPC in June 2013. Heart Rhythm. 2013;10(12):1932-63.

7. Stang A. Critical evaluation of the Newcastle-Ottawa scale for the assessment of the quality of nonrandomized studies in meta-analyses. Eur J Epidemiol. 2010;25(9):603-5.

8. Kuriachan VP, Sumner GL, Mitchell LB. Sudden cardiac death. Curr Probl Cardiol. 2015;40(4):133-200.

9. Freeman MF, Tukey JW. Transformations Related to the Angular and the Square Root. Ann Math Statist. 1950;21(4):607-11.

10. DerSimonian R, Laird N. Meta-analysis in clinical trials. Control Clin Trials. 1986;7(3):177-88.

11. Stijnen T, Hamza TH, Ozdemir P. Random effects meta-analysis of event outcome in the framework of the generalized linear mixed model with applications in sparse data. Stat Med. 2010;29(29):3046-67.

12. Debray TPA, Moons KGM, Riley RD. Detecting small-study effects and funnel plot asymmetry in metaanalysis of survival data: A comparison of new and existing tests. Research synthesis methods. 2018;9(1):4150 .

13. Patsopoulos NA, Evangelou E, Ioannidis JP. Sensitivity of between-study heterogeneity in meta-analysis: proposed metrics and empirical evaluation. Int J Epidemiol. 2008;37(5):1148-57.

14. Andorin A, Behr ER, Denjoy I, Crotti L, Dagradi F, Jesel L, et al. Impact of clinical and genetic findings on the management of young patients with Brugada syndrome. Heart Rhythm. 2016;13(6):1274-82.

15. Benito B, Sarkozy A, Mont L, Henkens S, Berruezo A, Tamborero D, et al. Gender differences in clinical manifestations of Brugada syndrome. J Am Coll Cardiol. 2008;52(19):1567-73.

16. Chinushi M, Komura S, Izumi D, Furushima H, Tanabe Y, Washizuka T, et al. Incidence and initial characteristics of pilsicainide-induced ventricular arrhythmias in patients with Brugada syndrome. Pacing Clin Electrophysiol. 2007;30(5):662-71. 
17. Delise P, Allocca G, Marras E, Giustetto C, Gaita F, Sciarra L, et al. Risk stratification in individuals with the Brugada type 1 ECG pattern without previous cardiac arrest: usefulness of a combined clinical and electrophysiologic approach. Eur Heart J. 2011;32(2):169-76.

18. Giustetto C, Drago S, Demarchi PG, Dalmasso P, Bianchi F, Masi AS, et al. Risk stratification of the patients with Brugada type electrocardiogram: a community-based prospective study. Europace. 2009;11(4):507-13.

19. Hernandez-Ojeda J, Arbelo E, Borras R, Berne P, Tolosana JM, Gomez-Juanatey A, et al. Patients With Brugada Syndrome and Implanted Cardioverter-Defibrillators: Long-Term Follow-Up. J Am Coll Cardiol. 2017;70(16):1991-2002.

20. Hiraoka M, Takagi M, Yokoyama Y, Sekiguchi Y, Aihara N, Aonuma K, et al. Prognosis and risk stratification of young adults with Brugada syndrome. J Electrocardiol. 2013;46(4):279-83.

21. Kamakura S, Ohe T, Nakazawa K, Aizawa Y, Shimizu A, Horie M, et al. Long-term prognosis of probands with Brugada-pattern ST-elevation in leads V1-V3. Circ Arrhythm Electrophysiol. 2009;2(5):495-503.

22. Kharazi A, Emkanjoo Z, Alizadeh A, Nikoo MH, Jorat MV, Sadr-Ameli MA. Mid-term follow-up of patients with Brugada syndrome following a cardioverter defibrillator implantation: a single center experience. Indian Pacing Electrophysiol J. 2007;7(1):33-9.

23. Ohkubo K, Watanabe I, Takagi Y, Okumura Y, Ashino S, Kofune M, et al. Electrocardiographic and electrophysiologic characteristics in patients with Brugada type electrocardiogram and inducible ventricular fibrillation: single center experience. Circ J. 2007;71(9):1437-41.

24. Ouali S, Boughzela E, Haggui A, Haouala H, Battikh K, Ben Ameur Y, et al. Clinical and electrophysiological profile of Brugada syndrome in the Tunisian population. Pacing Clin Electrophysiol. 2011;34(1):47-53.

25. Priori SG, Gasparini M, Napolitano C, Della Bella P, Ottonelli AG, Sassone B, et al. Risk stratification in Brugada syndrome: results of the PRELUDE (PRogrammed ELectrical stimUlation preDictive valuE) registry. J Am Coll Cardiol. 2012;59(1):37-45.

26. Schukro C, Berger T, Stix G, Pezawas T, Kastner J, Hintringer F, et al. Regional prevalence and clinical benefit of implantable cardioverter defibrillators in Brugada syndrome. Int J Cardiol. 2010;144(2):191-4.

27. Conte G, Sieira J, Sarkozy A, de Asmundis C, Di Giovanni G, Chierchia GB, et al. Life-threatening ventricular arrhythmias during ajmaline challenge in patients with Brugada syndrome: incidence, clinical features, and prognosis. Heart Rhythm. 2013;10(12):1869-74.

28. Sieira J, Conte G, Ciconte G, Chierchia GB, Casado-Arroyo R, Baltogiannis G, et al. A score model to predict risk of events in patients with Brugada Syndrome. Eur Heart J. 2017;38(22):1756-63.

29. Son MK, Byeon K, Park SJ, Kim JS, Nam GB, Choi KJ, et al. Prognosis after implantation of cardioverter-defibrillators in Korean patients with Brugada syndrome. Yonsei Med J. 2014;55(1):37-45.

30. Ueoka A, Morita H, Watanabe A, Morimoto Y, Kawada S, Tachibana M, et al. Prognostic Significance of the Sodium Channel Blocker Test in Patients With Brugada Syndrome. J Am Heart Assoc. 2018;7(10).

31. Sieira J, Brugada P. Management of Brugada Syndrome 2016: Should All High Risk Patients Receive an ICD? All High-Risk Patients Should Receive an Implantable Cardiac Defibrillator. Circulation Arrhythmia and electrophysiology. 2016;9(11).

32. Casado-Arroyo R, Berne P, Rao JY, Rodriguez-Manero M, Levinstein M, Conte G, et al. Long-Term Trends in Newly Diagnosed Brugada Syndrome: Implications for Risk Stratification. Journal of the American College of Cardiology. 2016;68(6):614-23.

33. Miyazaki T, Mitamura H, Miyoshi S, Soejima K, Aizawa Y, Ogawa S. Autonomic and antiarrhythmic drug modulation of ST segment elevation in patients with Brugada syndrome. J Am Coll Cardiol. 
1996;27(5):1061-70.

34. Brugada R, Brugada J, Antzelevitch C, Kirsch GE, Potenza D, Towbin JA, et al. Sodium channel blockers identify risk for sudden death in patients with ST-segment elevation and right bundle branch block but structurally normal hearts. Circulation. 2000;101(5):510-5.

35. Cheung CC, Mellor G, Deyell MW, Ensam B, Batchvarov V, Papadakis M, et al. Comparison of Ajmaline and Procainamide Provocation Tests in the Diagnosis of Brugada Syndrome. JACC Clinical electrophysiology. 2019;5(4):504-12.

36. Sacher F, Probst V, Maury P, Babuty D, Mansourati J, Komatsu Y, et al. Outcome after implantation of a cardioverter-defibrillator in patients with Brugada syndrome: a multicenter study-part 2. Circulation. 2013;128(16):1739-47.

37. Dereci A, Yap SC, Schinkel AFL. Meta-Analysis of Clinical Outcome After Implantable CardioverterDefibrillator Implantation in Patients With Brugada Syndrome. JACC Clin Electrophysiol. 2019;5(2):141-8.

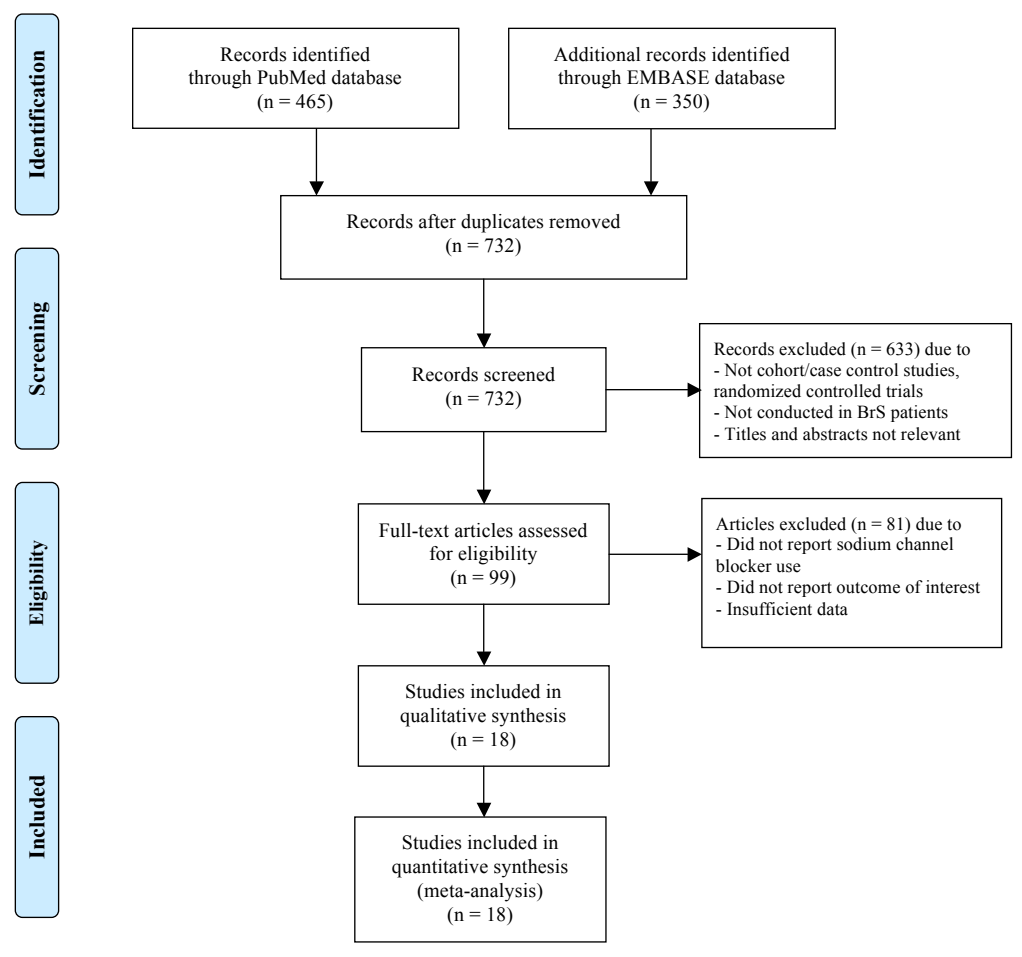

Figure 1 Search methodology and selection process 


\begin{tabular}{|c|c|c|c|}
\hline Study, year & & Incidence/1000 (95\% Cl) & \%Weight \\
\hline Andrion et al., 2016 & $\longrightarrow$ & $49.4(25.2,94.4)$ & 1.37 \\
\hline Benito et al., 2008 & $\rightarrow$ & $31.5(21.1,46.9)$ & 8.00 \\
\hline Chinushi et al., 2007 & $\rightarrow$ & $26.7(4.7,136.6)$ & 0.58 \\
\hline Delise et al., 2011 & $\rightarrow$ & $25.7(15.6,41.9)$ & 7.86 \\
\hline Giustetto C et al, 2017 & $\leftarrow$ & $13.9(6.7,28.4)$ & 11.24 \\
\hline Hernandez-Ojeda et al., 2017 & $\rightarrow-$ & $25.6(15.6,41.8)$ & 7.89 \\
\hline Hiraoka et al., 2013 & $\longrightarrow$ & $38.8(17.9,82.0)$ & 1.64 \\
\hline Kamakura et al., 2009 & $\rightarrow$ & $22.8(14.1,36.8)$ & 9.95 \\
\hline Kharazi et al, 2006 & & $0.0(0.0,357.6)$ & 0.05 \\
\hline Onkubo et al, 2007 & $\rightarrow$ & $21.4(3.8,111.6)$ & 0.90 \\
\hline Ouali et al., 2010 & $\rightarrow-$ & $22.7(4.0,118.1)$ & 0.80 \\
\hline Priori et al., 2012 & $\rightarrow$ & $27.2(15.9,45.9)$ & 6.35 \\
\hline Probst et al, 2010 & $\leftarrow$ & $25.3(18.0,35.5)$ & 14.19 \\
\hline Schukro et al., 2010 & +0 & $38.3(10.6,129.2)$ & 0.57 \\
\hline Sieira et al., 2017 & $\rightarrow$ & $29.6(17.0,51.0)$ & 5.12 \\
\hline Son et all, 2014 & 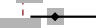 & $53.2(30.7,90.7)$ & 1.76 \\
\hline Ueoka et al, 2018 & $\leftarrow$ & $16.5(11.4,23.7)$ & 21.72 \\
\hline Overall $\left(\Lambda^{\prime 2}=14.7 \%, \mathrm{p}=0.3\right)$ & 0 & $23.8(19.8,27.8)$ & 100.00 \\
\hline
\end{tabular}

\begin{tabular}{|c|c|c|c|}
\hline Study, year & & Incidence/1000 (95 Cl) & $\%$ Weight \\
\hline Andrion et al., 2016 & $\leftarrow$ & $6.3(1.7,22.9)$ & 6.16 \\
\hline Benito et al., 2008 & $\leftarrow$ & $9.8(5.5,17.4)$ & 10.34 \\
\hline Chinushi et al., 2007 & -4 & $14.8(2.6,79.3)$ & 0.78 \\
\hline Delise et al., 2011 & $\leftarrow$ & $4.2(1.1,15.0)$ & 10.35 \\
\hline Giustetto C et al., 2017 & $\leftarrow$ & $3.9(1.1,14.2)$ & 10.94 \\
\hline Hernandez-Ojeda et al., 2017 & $\rightarrow$ & $15.7(7.2,33.9)$ & 3.56 \\
\hline Hiraoka et al., 2013 & $\rightarrow$ & $21.4(5.9,74.6)$ & 0.75 \\
\hline Kamakura et al., 2009 & $\leftarrow$ & $10.3(3.5,29.8)$ & 4.04 \\
\hline Kharazi et al., 2006 & & $96.6(26.9,292.6)$ & 0.04 \\
\hline Ohkubo et al., 2007 & $\leftarrow$ & $0.0(0.0,54.8)$ & 1.48 \\
\hline Ouali et al., 2010 & 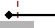 & $0.0(0.0,303.9)$ & 0.04 \\
\hline Priori et al., 2012 & $\leftarrow$ & $2.6(0.5,14.6)$ & 11.59 \\
\hline Probst et al., 2010 & $\leftarrow$ & $12.5(8.0,19.5)$ & 10.60 \\
\hline Schukro et al., 2010 & 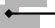 & $0.0(0.0,51.2)$ & 1.69 \\
\hline Sieira et al., 2017 & - & $3.6(1.6,7.8)$ & 16.77 \\
\hline Son et al., 2014 & & $62.1(30.4,122.7)$ & 0.33 \\
\hline Ueoka et all, 2018 & $\leftarrow$ & $5.0(1.7,14.6)$ & 10.56 \\
\hline Overall $(1 / 2=36.7 \%, p=0.1)$ & 0 & $6.5(3.9,9.1)$ & 100.00 \\
\hline
\end{tabular}




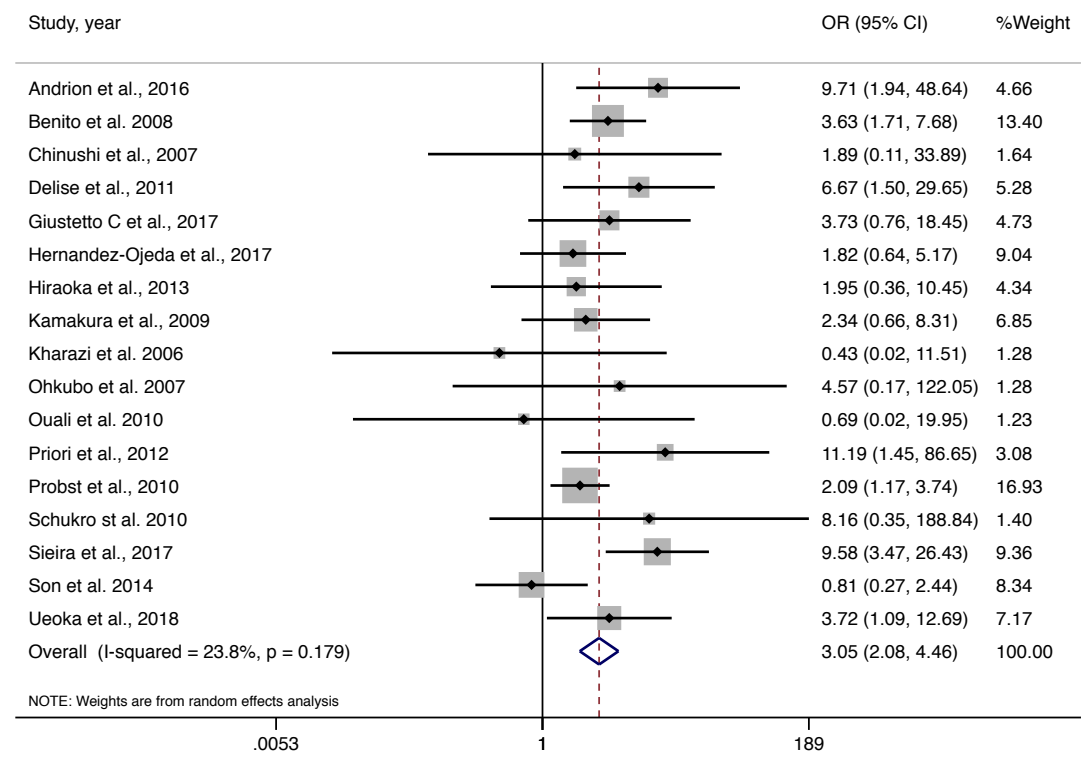

Study, year $\quad$ OR $(95 \% \mathrm{Cl}) \quad \%$ Weight

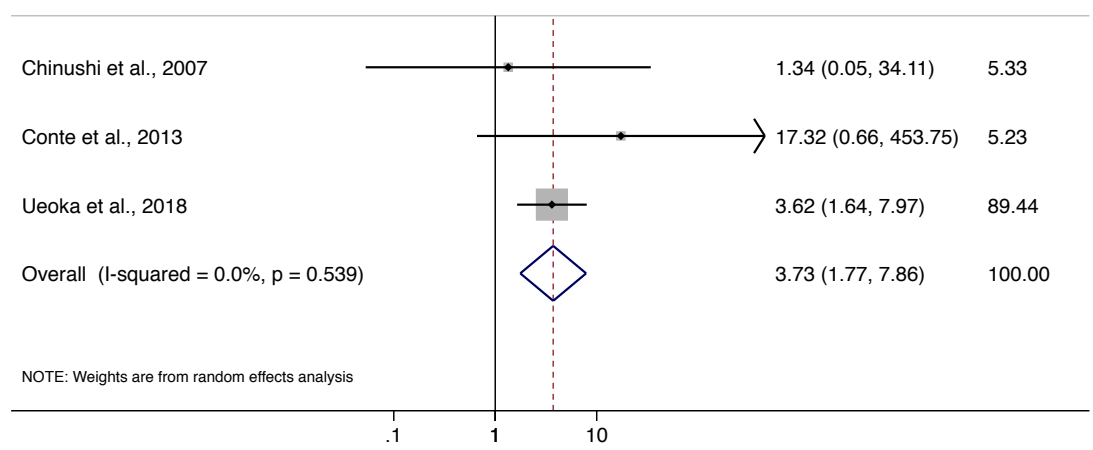




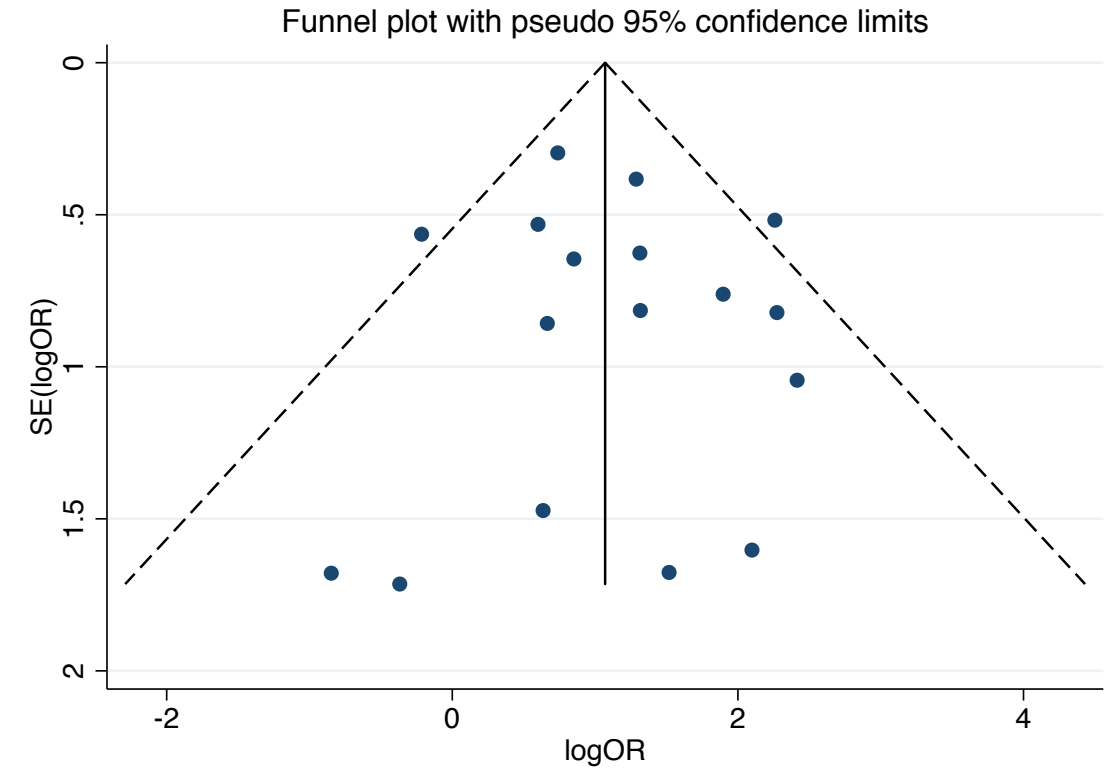

